\title{
Side Chain Mediated Electronic Contact between a Tetrahydro-4H- thiopyran-4-ylidene-Appended Polythiophene and CdTe Quantum Dots
}

\author{
Rick van Beek, ${ }^{[a]}$ Arjan P. Zoombelt, ${ }^{[a]}$ Leonardus W. Jenneskens, ${ }^{[a]}$ \\ Cornelis A. van Walree, ${ }^{*[a]}$ Celso de Mello Donegá, ${ }^{[b]}$ Dirk Veldman, ${ }^{[c]}$ and \\ René A. J. Janssen ${ }^{[c]}$
}

\begin{abstract}
The properties of a mixed CdTe quantum dot/tetrahydro- $4 H$-thiopyran-4-ylidene-functionalized polythiophene system are reported. This system was prepared by exposing trioctylphosphine (TOP)-capped CdTe quantum dots to the polythiophene in solution. Strong fluorescence emission quenching and shortening of the fluorescence emission lifetimes of both the
\end{abstract}

polythiophene and the quantum dots occur when they are mixed, indicating the occurrence of photoinduced charge separation. Photoinduced absorption

Keywords: electron transfer photophysics - polythiophenes . quantum dots $\cdot$ semiconductors spectroscopy reveals a considerable decrease in the population of the polythiophene triplet excited state in the mixed system. These results demonstrate that between the quantum dots and the polythiophene there is both physical and electronic contact, which is mediated by the tetrahydro- $4 H$-thiopyran-4-ylidene side chains.

\section{Introduction}

Hybrid inorganic/organic semiconducting systems are promising materials for application in optoelectronic devices, such as photovoltaic systems ${ }^{[1-5]}$ and light-emitting diodes (LEDs). ${ }^{[5-10]}$ As inorganic constituent, nanocrystalline inorganic semiconductors (quantum dots) form an interesting class of materials because of their size-tunable photophysical properties $^{[11-17]}$ and high luminescence quantum yields. ${ }^{[13,14,18-22]}$ In addition, quantum dots can be relatively easily prepared by wet-chemical methods, ${ }^{[12,19,23,24]}$ whereas the synthesis of bulk inorganic semiconductors often requires high temperatures or very low pressures. ${ }^{[25,26]}$

[a] Dr. R. van Beek, A. P. Zoombelt, Prof. Dr. L. W. Jenneskens, Dr. C. A. van Walree

Debye Institute, Organic Chemistry and Catalysis, Utrecht University Padualaan 8, $3584 \mathrm{CH}$ Utrecht (The Netherlands)

Fax: $(+31) 30-253-4533$

E-mail: c.a.vanwalree@chem.uu.nl

[b] Dr. C. de Mello Donegá

Debye Institute, Condensed Matter and Interfaces, Utrecht Universi-

Princetonplein 1, 3584 CC Utrecht (The Netherlands)

[c] D. Veldman, Prof. Dr. R. A. J. Janssen

Laboratory for Macromolecular and Organic Chemistry, Eindhoven University of Technology

P.O. Box 513, $5600 \mathrm{MB}$ Eindhoven (The Netherlands)
Quantum dots are usually capped with a layer of organic molecules to prevent clustering of particles and to suppress nonradiative decay of excited states at the quantum dot surface. ${ }^{[12,19,20,24,27-29]}$ Unfortunately, this capping layer forms an obstacle to the application of quantum dots in hybrid inorganic/organic semiconducting systems. Owing to its electronically insulating character, it prevents electronic contact between quantum dots and other electroactive materials. Nevertheless, hybrid quantum dot/organic systems for optoelectronic applications have been obtained by casting from solutions of mixtures of both constituents ${ }^{[1-3,30]}$ or by deposition techniques. ${ }^{[7-10]}$ Although encouraging results, such as improved electroluminescence efficiencies compared with onecomponent systems and the occurrence of photoinduced charge separation, have been reported, the development of hybrid quantum dot/organic systems with a substantial electronic coupling between the constituents is desired. This requires optimized contact, both physical and electronic, between the components. One way to achieve this is to functionalize a polymer with side chains possessing a high affinity for inorganic materials. ${ }^{[31,32]}$ Thus the polymer can substitute for the original capping layer on quantum dots and passivate the surface of quantum dots. More importantly, the side chain must also be able to mediate charge transport between the quantum dots and the polymer backbone.

It has been shown that tetrahydro- $4 H$-thiopyran-4-ylidene-functionalized compounds make electronic contact 
with CdSe quantum dots and mediate charge transport between the quantum dots and a gold electrode ${ }^{[33]}$ or between two gold electrodes. ${ }^{[34,35]}$ This charge transport is relayed by $\sigma-\pi$ through-bond interactions. ${ }^{[36,37]}$ Consequently, side chains based on this moiety are promising candidates to achieve both physical and electronic contact between a semiconducting polymer and inorganic semiconductor quantum dots. Therefore, we have investigated whether it is possible to substitute (part of) the cap-

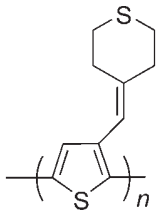

poly(4) ping layer of CdTe quantum dots with a polythiophene $(\operatorname{poly}(4))$ bearing a tetrahydro- $4 \mathrm{H}$ thiopyran-4-ylidene group in the side chain and whether this side chain induces substantial electronic contact between the polymer and the quantum dot. CdTe quantum dots were used particularly because of their relatively low bandgap $(1.5 \mathrm{eV}$ for bulk materi$\left.\mathrm{al}^{[38]}\right)$, which enables the separation of the fluorescence emission bands of the quantum dots and the polythiophene. The complexation between the quantum dots and the polymer, and the optical properties of the hybrid systems, were investigated with UV/Vis absorption, fluorescence, and photoinduced absorption (PIA) spectroscopy.

\section{Results and Discussion}

Synthesis: Monomer 4 was synthesized in a two-step procedure (Scheme 1). Tetrahydro-4H-thiopyran-4-carboxylic acid 1 was doubly deprotonated and subsequently coupled to 2,5dibromothiophene carboxaldehyde 2 to yield $\beta$-hydroxy acid
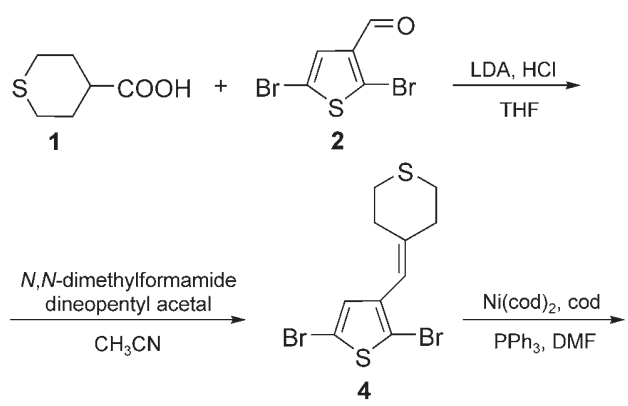

Scheme 1 . Synthetic route to poly(4).

3. ${ }^{[39]}$ Decarboxylative dehydration of 3 with $N, N$-dimethylformamide dineopentyl acetal furnished alkene 4. ${ }^{[39]} \mathrm{Al}-$ though 3 was obtained with a purity of only $35 \%$, this did not prevent the formation and isolation of pure $\mathbf{4}$ in the next step; it was obtained in $19 \%$ yield relative to the starting aldehyde 2. Monomer 4 was polymerized using [Ni$\left.(\operatorname{cod})_{2}\right]$, furnishing poly $(4),{ }^{[40]}$ which was soluble in chloroform and partially soluble in dichloromethane and THF. As determined by size-exclusion chromatography (SEC) the weight-average molecular weight $M_{\mathrm{w}}$ of poly(4) was $2.7 \times 10^{3}$ with a polydispersity $D$ of 1.4 . The presence of two olefinic ${ }^{1} \mathrm{H}$ NMR signals of similar intensity indicates that poly(4) has a highly random regioregularity.

CdTe quantum dots were obtained by a procedure based on that developed by Wuister et al. ${ }^{[12,20]}$ To facilitate complexation with poly(4), the excess trioctylphosphine/dodecylamine (TOP/DDA) capping agent was removed by precipitation into methanol. ${ }^{1} \mathrm{H}$ NMR spectroscopy revealed almost complete removal of DDA, implying that the precipitated quantum dots were covered predominantly with TOP.

Properties of poly(4): The UV/Vis absorption maximum of poly(4) in chloroform solution is situated at $477 \mathrm{~nm}$ (Figure 1A). It is shifted bathochromically with respect to the solution absorption maxima generally observed for regiorandom poly(3-alkylthiophene)s, which lie near $430 \mathrm{~nm} .^{[41,42]}$ The relatively low energy of the absorption maximum of poly(4) is explained by the extension of the conjugated system of the repeating unit with the olefinic bond of the tetrahydro- $4 H$-thiopyran-4-ylidene substituent. The absorption maximum of poly(4) as a film on quartz (Figure 1A) is at a somewhat longer wavelength $(500 \mathrm{~nm})$ than in solution.
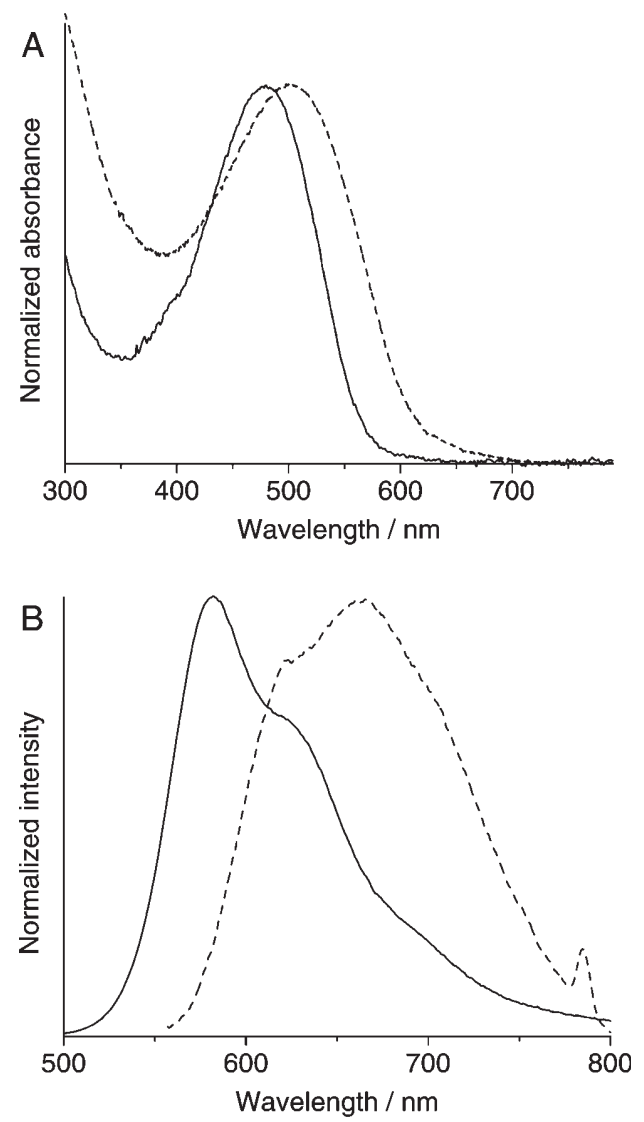

Figure 1. A) UV/Vis absorption spectra and B) fluorescence emission spectra of poly(4) in chloroform solution (solid line) and as a film on quartz, cast from chloroform solution (broken line). In B, $\lambda_{\mathrm{exc}}=460 \mathrm{~nm}$ for the spectrum in solution and $\lambda_{\mathrm{exc}}=500 \mathrm{~nm}$ for the spectrum of the film. 
This is the consequence of a better ordering of the polymer chains in the solid state, which leads to a longer conjugation length. ${ }^{[41,43]}$

The fluorescence emission spectrum of poly(4) in chloroform solution (Figure 1B) has a maximum at $582 \mathrm{~nm}$ and a shoulder near $630 \mathrm{~nm}$, representing a vibronic transition. The emission spectrum of the film on quartz is red-shifted, with the maximum now at $665 \mathrm{~nm}$ (Figure 1B). The red shift upon going from solution to the solid state $\left(2200 \mathrm{~cm}^{-1}\right)$ is considerably stronger than that found with absorption spectroscopy $\left(800 \mathrm{~cm}^{-1}\right)$. This is explained by the occurrence of interchain energy transfer in the solid state; in the solid state longer chain segments are involved in the fluorescence process than in the absorption process.

The properties of poly $(4)$ were investigated further with cyclic voltammetry (CV) in THF solution. Anodic scans revealed a single irreversible oxidation wave centered at $1.0 \mathrm{~V}$ versus SCE, whereas cathodic scans exhibited a single irreversible reduction wave centered at $-1.7 \mathrm{~V}$ versus SCE. The oxidation potential is more positive than that found for poly[3-(2,5,8-trioxanonyl)thiophene] (PTT) in THF $(0.8 \mathrm{~V}$ versus $\left.\mathrm{SCE}^{[43,44]}\right)$. The relatively high oxidation potential of poly(4), which may be the consequence of a higher degree of distortion of the polythiophene backbone by the bulky ring-containing substituents, seems to be in disagreement with the observation that the absorption maximum of poly(4) (465 nm for a saturated solution in THF) is situated at a longer wavelength than that of PTT $(440 \mathrm{~nm}$ in $\left.\mathrm{THF}^{[43]}\right)$. This apparent contradiction can be explained only by assuming that the effect of the tetrahydro- $4 H$-thiopyran4-ylidene substituent on the electronic spectrum of poly(4) is based predominantly on a stabilization of the conduction band.

Mixed CdTe quantum dot/poly(4) systems: Hybrid CdTe quantum dot/poly(4) systems were prepared in such a way that at most about $40 \%$ of the quantum dot surface could be occupied by poly(4). This corresponds with a CdTe unit/ poly(4) repeating unit ratio of approximately 9:1 (see Experimental Section). The CdTe quantum dots used in these studies had an average particle diameter of $4.0 \mathrm{~nm}$, as deduced from the first absorption maximum near $620 \mathrm{~nm}^{\left[{ }^{[1]}\right.}$

Figure $2 \mathrm{~A}$ shows the UV/Vis absorption spectrum of the mixed system in chloroform, together with the spectra of pristine poly(4) and bare TOP-capped CdTe quantum dots. The concentrations of CdTe quantum dots $\left(9.8 \times 10^{-5} \mathrm{~mol}\right.$ CdTe units $\left.\mathrm{L}^{-1}\right)$ and $\operatorname{poly}(4)\left(1.1 \times 10^{-5} \mathrm{~mol}\right.$ repeating units $\mathrm{L}^{-1}$ ) in the mixed sample were equal to those in the solutions of the separate components. It is readily seen from the region above $600 \mathrm{~nm}$ that the CdTe quantum dot absorption remains unaffected by exposure to poly(4). However, a difference spectrum, resulting from subtraction of the spectrum of the bare CdTe quantum dots from that of the mixed system, shows a poly(4) absorption that is slightly red-shifted (with the maximum now at $490 \mathrm{~nm}$ ) and broadened compared with that of pristine poly(4). This could be an indication that a change in backbone conformation of poly(4) has
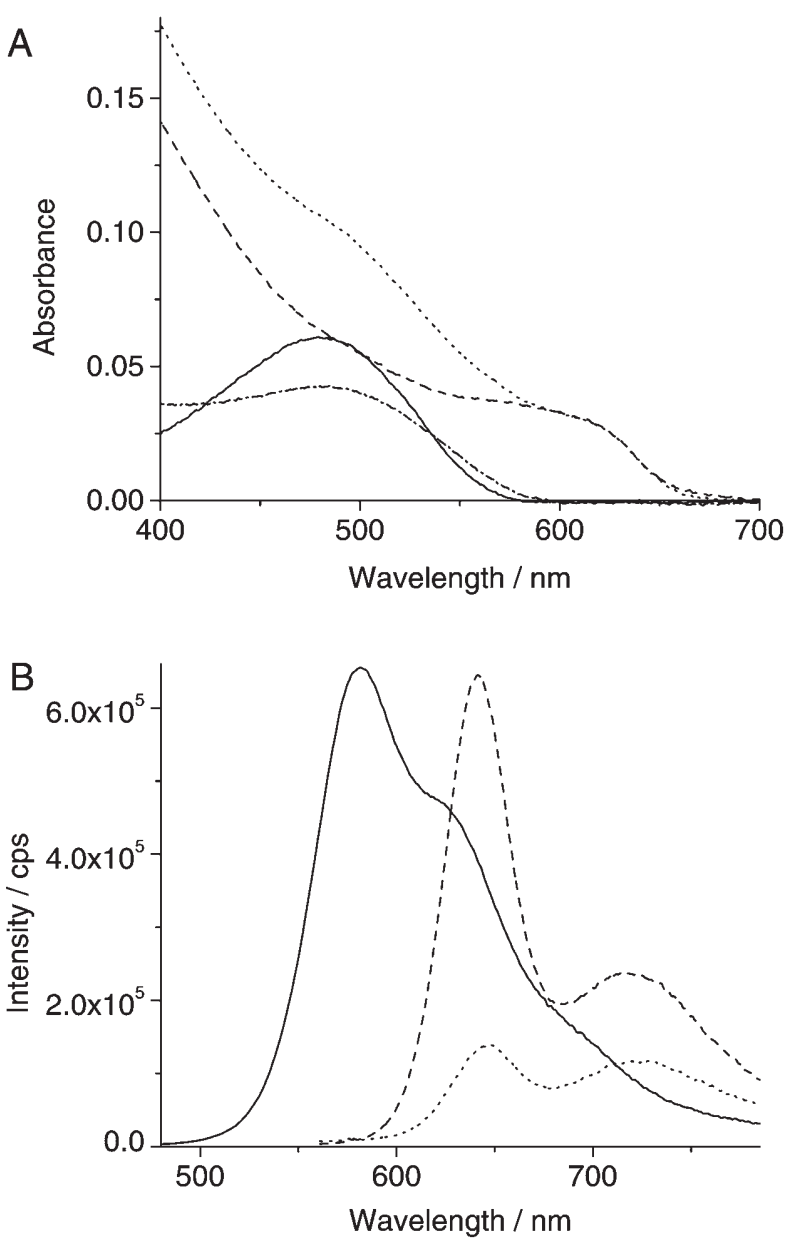

Figure 2. A) UV/Vis absorption spectra and B) emission spectra $\left(\lambda_{\mathrm{exc}}=\right.$ $406 \mathrm{~nm}$ ) of pristine poly(4) (solid line), CdTe quantum dots with TOP capping (broken line) and in the presence of $40 \%$ poly(4) (dotted line), recorded in chloroform solution. A also shows a difference spectrum (dash-dotted line) obtained by subtraction of the spectrum of the TOPcapped CdTe quantum dots from that of the mixed system.

occurred upon mixing with the quantum dots. It is very possible that the change in backbone conformation is induced by adhesion of poly(4) to the surface of the quantum dots. A similar phenomenon has been found for PTT deposited on a $\mathrm{ZnSe}$ single-crystal surface. ${ }^{[43]}$

The emission spectrum of the mixture, along with the spectra of the individual components, is shown in Figure 2B. The spectra were recorded upon excitation at $406 \mathrm{~nm}$, which implies that in the mixed sample both components were excited. As with absorption measurements, the concentrations of both components in the mixed sample equalled those in the samples containing the separate components. They were $1.6 \times 10^{-5} \mathrm{~mol} \mathrm{CdTe}$ units $\mathrm{L}^{-1}$ and $1.9 \times 10^{-6} \mathrm{~mol}$ poly(4) repeating units $\mathrm{L}^{-1}$. The emission maximum of pristine poly(4) is at $582 \mathrm{~nm}$ while the bare CdTe quantum dots give an exciton emission maximum at $645 \mathrm{~nm}$ and a trap emission centered at $720 \mathrm{~nm}$. The latter originates from defect states that are populated by nonradiative decay from the exciton state (that is, exciton trapping). It is conspicuous that in the mixed system the poly(4) emission has almost completely 
disappeared (Figure 2B). Furthermore, the intensity at the maximum of the CdTe quantum dot exciton emission has decreased by a factor of approximately 4.5 , while that at the maximum of the defect emission has decreased by a factor of about 2 . This strong fluorescence quenching shows that a new, efficient decay channel has been created upon mixing poly(4) with CdTe quantum dots. It is likely that this new decay channel involves formation of a charge-separated (CS) state. Thus, after population of an excited state, which can be localized either on the quantum dots or on poly(4), electron transfer from poly(4) to the CdTe quantum dots, or vice versa, may occur, causing quenching of the local poly(4) and CdTe quantum dot fluorescence. The observation that quenching of the defect emission of the quantum dots is less pronounced than that of the exciton emission shows that the radiationless transition to the defect state suffers less from introduction of the charge separation pathway than radiative decay from the exciton state. This is consistent with the trapping process being faster than the radiative decay (vide infra). Most importantly, the strong fluorescence quenching implies that attachment of poly(4) at the quantum dot surface has taken place.

To substantiate the findings from the steady-state fluorescence spectra, fluorescence decay curves were measured for the mixed system and the individual components. The fluorescence decay was monitored at $\lambda_{\mathrm{em}}=582$ and $645 \mathrm{~nm}$, the respective maxima of the poly(4) and CdTe quantum dot exciton emission. The fluorescence decay of pristine poly(4) is mono-exponential (Figure $3 \mathrm{~A}$ ) and has a lifetime of $0.48 \mathrm{~ns}$, a common value for a polythiophene in solution. ${ }^{[45,46]}$ In the mixed sample the decay is multi-exponential and much faster than for pristine poly(4). The decay time of the fastest component is estimated to be 70 ps or faster (limited by the temporal resolution of the experimental setup at $10 \mathrm{MHz}$ repetition rate; see Experimental Section). The considerably faster fluorescence decay in the mixed sample confirms the findings from the fluorescence emission spectra: in presence of CdTe quantum dots a new route for depopulation of the singlet excited state of poly(4) is created, causing quenching of the local fluorescence. The decay curve of the mixed system also reveals a slower component with a decay time of $5.0 \mathrm{~ns}$ and an amplitude that is approximately two orders of magnitude lower than that of the fast component (vide infra).

In Figure 3B fluorescence decay curves of the mixed sample and of the pristine CdTe quantum dots, monitored at the maximum exciton emission $(645 \mathrm{~nm})$, are depicted. The decay curves are multi-exponential, reflecting a distribution of fluorescence lifetimes as a result of sample inhomogeneities (both in quantum dot size and in surface defect structure). ${ }^{[12]}$ Despite the multi-exponential nature of the decay curve, for the pristine CdTe quantum dots it could be fitted with a bi-exponential function, giving an average fast decay component of $0.9 \mathrm{~ns}$ and an average slower decay component of $11 \mathrm{~ns}$. The short exciton lifetimes reflect the decay in nanocrystals where exciton trapping predominates, ${ }^{[12]}$ while long exciton lifetimes originate from quantum dots in which
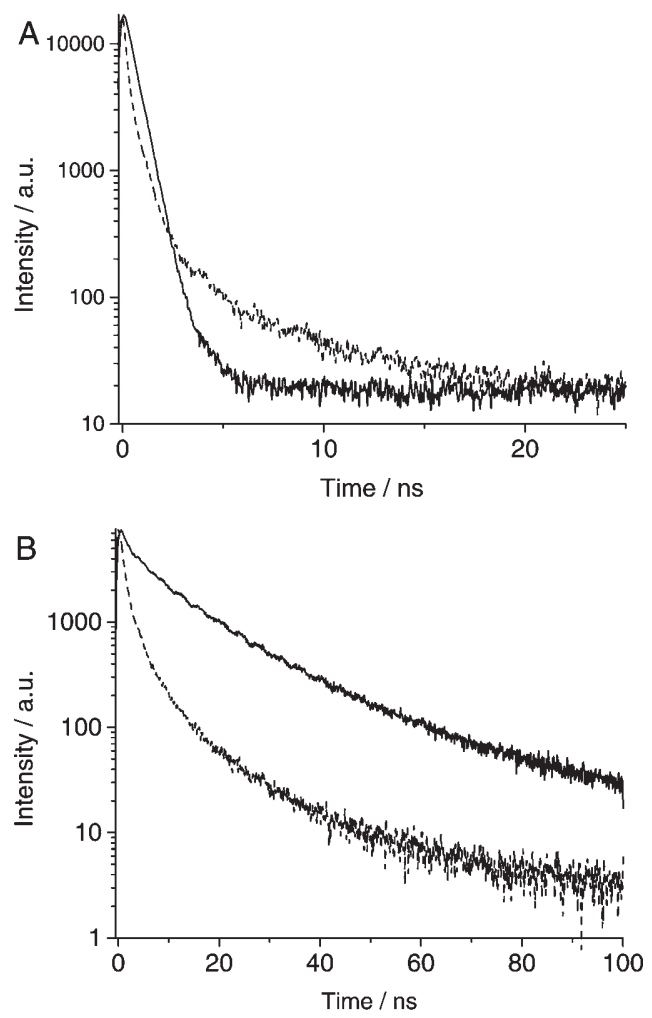

Figure 3. A) Fluorescence decay curves (chloroform solution) of pristine poly(4) (solid line) and a poly(4)/CdTe quantum dot mixture (broken line), monitored at $\lambda_{\mathrm{exc}}=406 \mathrm{~nm}$ and $\lambda_{\mathrm{em}}=582 \mathrm{~nm}$. B) Fluorescence decay curves (chloroform solution) of pristine CdTe quantum dots (solid line) and CdTe quantum dots capped with $40 \%$ poly(4) (broken line), monitored at $\lambda_{\mathrm{exc}}=406 \mathrm{~nm}$ and $\lambda_{\mathrm{em}}=645 \mathrm{~nm}$. The traces are not deconvoluted from the instrumental response function.

radiative decay prevails. Bi-exponential fitting of the decay curve of the mixed system yields a main component with a very fast decay time ( $\leq 120 \mathrm{ps}$, limited by the temporal response of the instrumental setup at $2.5 \mathrm{MHz}$ repetition rate; see Experimental Section) and a component with a longer average decay time of $7.1 \mathrm{~ns}$. The short component again shows that the exciton decay in the mixed system is much faster than in the pristine quantum dots. This implies that the charge transfer process (rate $\geq 7 \times 10^{9} \mathrm{~s}^{-1}$ as derived from the fast decay component in the mixed system) competes with the trapping process in most of the quantum dots (rate $\approx 1 \times 10^{9} \mathrm{~s}^{-1}$, derived from the fast decay component of the pristine quantum dots) and is much faster than the exciton radiative decay. Similar fast charge transfer rates can be derived from the fluorescence decay curves of poly(4) in the mixed system $\left(\geq 1 \times 10^{10} \mathrm{~s}^{-1}\right)$. The $7.1 \mathrm{~ns}$ lifetime is comparable to the long component of pristine quantum dots and probably originates from a small fraction of free quantum dots. The $5.0 \mathrm{~ns}$ component of the mixed system measured at $\lambda_{\mathrm{em}}=582 \mathrm{~nm}$ (Figure $3 \mathrm{~A}$ ) is also thought to originate from a small fraction of free quantum dots which were excited at $406 \mathrm{~nm}$ and emit at $582 \mathrm{~nm}$.

Photoinduced absorption spectroscopy was performed to gain further insight into the photophysical behavior of the 
mixed CdTe quantum dot/poly(4) system. The PIA spectrum of pristine poly(4) (Figure 4, solid line) shows one positive band centered at $1.42 \mathrm{eV}(\lambda=875 \mathrm{~nm})$, which corresponds to a transition from a triplet exciton $\left(\mathrm{T}_{1}\right)$ to a higher triplet

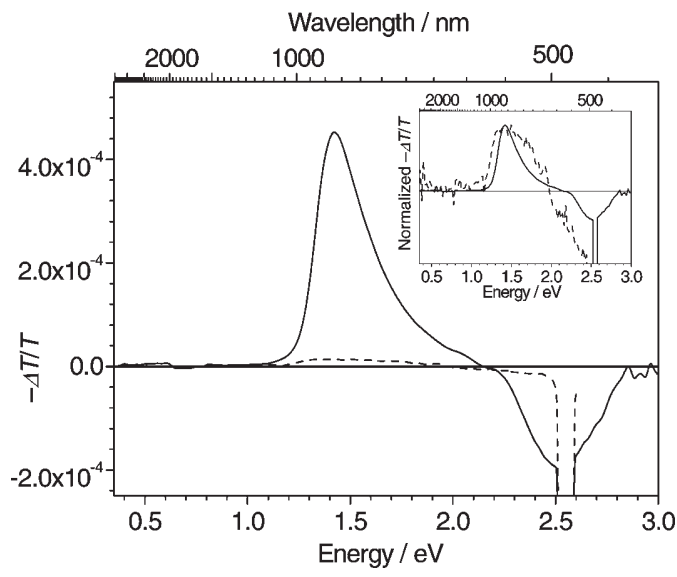

Figure 4. Photoinduced absorption spectra of pristine poly(4) (solid line) and poly(4) deposited onto CdTe quantum dots (broken line), measured in chloroform solution. The poly(4) concentration is equal in both the pristine poly(4) sample and in the mixed system. Inset: normalized spectra.

state $\left(\mathrm{T}_{n}\right)$. Similar triplet-associated PIA signals have been observed for poly(3-alkylthiophene)s. ${ }^{[22,47-50]}$ At energies above $2.3 \mathrm{eV}$ the spectra show photoinduced ground-state bleaching. ${ }^{[42,50]}$ The PIA spectrum of the mixed system shows a $\mathrm{T}_{1}-\mathrm{T}_{n}$ signal that is slightly broadened compared with the signal of pristine poly(4), but is situated at similar energies (see insert in Figure 4, which shows the PIA spectra normalized at $1.4 \mathrm{eV}$ ). More importantly, the signal of the mixed system has a considerably (approximately 30 times) lower intensity. This implies that population of the $T_{1}$ state of poly(4) is largely prevented, which is in line with the existence of an extra decay channel in the mixed system. The marked decrease in poly(4) ground-state bleaching in the mixed system implies that almost no excited state species with a lifetime of $10 \mu \mathrm{s}-10 \mathrm{~ms}$ (which are typically monitored with the PIA setup) are present and that the system must have decayed back to the ground state in a shorter period. It is not very probable that the disappearance of the poly(4) $\mathrm{T}_{1}-\mathrm{T}_{n}$ absorption is caused by increased spin-orbit coupling due to the presence of heavy elements in the CdTe quantum dots. In that case quenching of the CdTe quantum dot emission upon mixing with poly(4) should not occur. Note that if the quenching of the $\mathrm{T}_{1}-\mathrm{T}_{n}$ absorption were the consequence of a CdTe-induced heavy atom effect, this would also present compelling evidence for the deposition of poly(4) at the quantum dot surface. In the PIA spectrum of the mixed system no signals belonging to polaron states of poly(4), which might be expected in the case of photoinduced charge separation in a mixed quantum dot/polymer system, ${ }^{[1,50]}$ can be discerned. Such polaron-associated PIA signals usually occur as two broad bands: one centered near
$1.5 \mathrm{eV}$ and another near $0.5 \mathrm{eV} \cdot^{[1,47,48,50-53]}$ It is likely that polaron states decay to the ground state on a shorter timescale than monitored with the PIA setup.

Whether photoinduced charge separation occurs can be assessed by considering the thermodynamics of the process using Equation (1). ${ }^{[54]}$ Moreover, this provides insight into which components of the hybrid CdTe quantum dot/poly(4) system function as the electron donor and as the electron acceptor.

$\Delta G^{0}=E_{\text {ox }}(\mathrm{D})-E_{\text {red }}(\mathrm{A})-E_{00}+C$

In Equation (1) $\Delta G^{0}$ is the driving force for photoinduced charge separation. $E_{\mathrm{ox}}(\mathrm{D})$ represents the oxidation potential of the donor, whereas $E_{\text {red }}(\mathrm{A})$ is the reduction potential of the acceptor. $E_{00}$ stands for the zero-zero excitation energy, that is, the smallest bandgap in the hybrid system, and $C$ is a solvent-dependent term. ${ }^{[54]}$ From the absorption onset of CdTe quantum dots (Figure 2A) $E_{00}$ was determined to be $1.9 \mathrm{eV}$. For poly (4) $E_{\text {ox }}=+1.0 \mathrm{~V}$ and $E_{\text {red }}=-1.7 \mathrm{~V}$ versus $\mathrm{SCE}$, as found with $\mathrm{CV}$ in THF (vide supra). For CdTe quantum dots various values of $E_{\text {ox }}$ and $E_{\text {red }}$ have been reported in the literature. In Table 1 , literature redox poten-

Table 1. $\Delta G^{0}$ of charge separation calculated using different sets of CdTe quantum dot redox potentials. ${ }^{[\mathrm{a}]}$

\begin{tabular}{|c|c|c|c|c|}
\hline Ref. & $\begin{array}{l}E_{\text {ox }}, \text { CdTe quan- } \\
\text { tum dots [V vs. } \\
\text { SCE] }\end{array}$ & $\begin{array}{l}E_{\text {red }}, \text { CdTe quan- } \\
\text { tum dots [V vs. } \\
\text { SCE] }\end{array}$ & $\begin{array}{l}\Delta G^{0} \\
(\operatorname{poly}(\mathbf{4})= \\
\text { donor) }[\mathrm{eV}]\end{array}$ & $\begin{array}{l}\Delta G^{0} \text { (poly }(\mathbf{4}) \\
=\text { acceptor) } \\
{[\mathrm{eV}]}\end{array}$ \\
\hline [38] & -0.2 & -2.1 & $+1.2+C$ & $-0.4+C$ \\
\hline [55] & +0.45 & -1.7 & $+0.8+C$ & $+0.25+C$ \\
\hline [56] & +0.3 & -1.1 & $+0.2+C$ & $+0.1+C$ \\
\hline
\end{tabular}

[a] The redox potentials listed refer to quantum dots with diameters of $3.6-4.0 \mathrm{~nm}$

tials of CdTe particles with diameters similar to that of the particles used in this study are given. ${ }^{[38,55,56]}$ The spread in CdTe quantum dot redox potentials is due to differences in measurement method (pulse radiolysis ${ }^{[38]}$ and cyclic voltammetry in either a $5: 1(\mathrm{v} / \mathrm{v})$ benzene/acetonitrile mixture ${ }^{[55]}$ or aqueous solution ${ }^{[56]}$ ). Furthermore, different capping agents (thioglycerol, ${ }^{[38]}$ trioctylphosphine oxide, ${ }^{[55]}$ or thioglycolic acid $\left.^{[56]}\right)$ were used. The values of $\Delta G^{0}$ with the different sets of CdTe quantum dot redox potentials were calculated both when poly(4) was assumed to be the electron donor and when it was taken to be the electron acceptor (Table 1).

It can be seen that $\Delta G^{0}$ is negative when using $E_{\mathrm{ox}}$ for CdTe quantum dots with a thioglycerol capping from pulse radiolysis ${ }^{[38]}$ and when taking poly(4) as acceptor. However, use of this $E_{\text {ox }}$ value is not entirely realistic since the negatively charged thiolate capping will shift the potential toward a value which is too negative for a (neutral) dialkyl sulfide capping. With the cyclic voltammetry data a slightly positive $\Delta G^{0}$ value has been calculated, again under the assumption that CdTe is the electron donor. For the quantum dots with trioctylphosphine oxide ligands the $E_{\text {ox }}$ value may however be somewhat too positive, since the organic shells 
form a kinetic barrier to electron removal from the quantum dot. ${ }^{[56]}$ We therefore expect that the actual $\Delta G^{0}$ will be close to zero (the constant $C$ is expected not to exceed a few tenths of an electronvolt in the present systems). This does not confirm unambiguously that photoinduced charge separation is allowed thermodynamically, but it also does not render it unlikely. It is conspicuous that $\Delta G^{0}$ is in general more negative when poly(4) is assumed to be the electron acceptor than when it is taken to be the donor. This result is surprising since in inorganic semiconductor/semiconducting polymer mixtures the polymer typically functions as an electron donor. ${ }^{[1,50]}$ The possibly reversed donor-acceptor behavior of the CdTe quantum $\operatorname{dot} / \operatorname{poly}(4)$ system is caused by the very high energies of the band edges of the CdTe quantum dots compared with other semiconductors. ${ }^{[16,57]}$

\section{General Discussion and Conclusions}

From the fluorescence spectra and decay times of the hybrid CdTe quantum dot/poly(4) systems it is evident that poly(4) has affinity for the surface of CdTe quantum dots. This shows that it is possible to deposit a semiconducting polymer on a quantum dot through interaction with the side chain. The observed fluorescence quenching and fluorescence lifetime shortening indicate that photoinduced charge transfer takes place. Thermodynamic considerations do not fully confirm the occurrence of photoinduced charge separation in mixed CdTe quantum dot/poly(4) systems, but they also do not contradict it. Surprisingly, the data tend to suggest that, if charge transfer takes place in the mixed system, CdTe quantum dots act as the electron donor and poly(4) acts as the electron acceptor.

PIA spectroscopy did not reveal polaron states in the mixed CdTe quantum dot/poly(4) system. This implies that, if polarons are present, their lifetime is short. In solution at room temperature, the lifetime of positively charged polarons may indeed be shorter than the microsecond-millisecond range, while negatively charged polarons (the formation of which is suggested by thermodynamic investigations) are expected to possess even shorter lifetimes, since these are less stable in a polythiophene. In addition, it cannot be excluded that trapping of charge carriers at defect sites on the quantum dots also contributes to their fast decay. This could be in line with the observation that the intensity of the CdTe quantum dot defect emission relative to the exciton emission intensity increases upon mixing with poly(4) (Figure 2B).

A schematic representation of the possible possible photophysical events in the mixed CdTe quantum dot/poly(4) system is shown in (Figure 5). Excitation of pristine TOPcapped quantum dots yields local exciton or defect emission. When isolated poly(4) is excited, $\mathrm{S}_{1}-\mathrm{S}_{0}$ fluorescence is observed. Intersystem crossing to the first triplet state $T_{1}$ occurs as well, giving rise to the $\mathrm{T}_{1}-\mathrm{T}_{n}$ triplet-triplet absorption. When poly(4) is brought into contact with the CdTe quantum dots, both the fluorescence and intersystem cross-

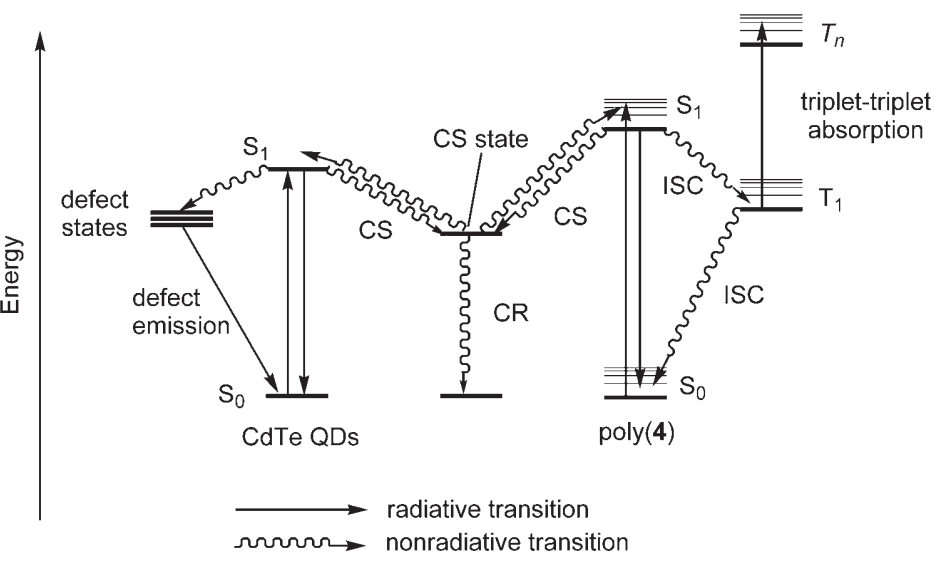

Figure 5. Schematic of photophysical transitions in the mixed CdTe quantum dot/poly(4) system. CS = charge separation; CR = charge recombination; ISC $=$ intersystem crossing. Arrows for nonradiative transitions to the ground state from the quantum dot exciton and defect states and the polymer $S_{1}$ and $T_{1}$ states have been omitted.

ing pathways are strongly suppressed. This is explained by rapid decay to a charge-separated state, which presumably is formed by transfer of an electron from the quantum dot to poly(4) ${ }^{[58]}$ In view of the observation that it is faster than the temporal resolution of the equipment used in the decay time measurements, the charge separation occurs with a rate of at least $10^{10} \mathrm{~s}^{-1}$. Rapid photoinduced charge separation in the mixed system also takes place upon excitation of the CdTe quantum dots, thereby largely preventing both $\mathrm{CdTe}$ exciton and defect emission. The most likely origin of the residual exciton emission and the 5-7 ns decay component is free (that is, TOP-capped) quantum dots. This would not be expected in view of the maximum coverage of $40 \%$, but can be explained by attachment of one polymer chain to a quantum dot facilitating attachment of other polymer chains. Greater insight into the surface coverage and the dynamics of the aggregation process can be obtained by SternVolmer-like studies.

In conclusion, both physical and electronic contact with CdTe quantum dots has been achieved by functionalizing a polythiophene with tetrahydro- $4 H$-thiopyran-4-ylidene side chains. It is noteworthy that fluorescence quenching was also found in blends of CdSe quantum dots and some poly ( $p$-phenylene vinylene) (PPV) derivatives. ${ }^{[1-3]}$ However, in these cases the inorganic and organic components were brought into physical contact by casting from a solution containing the two components. The particular significance of the results reported here is that electronic contact was created in solution, which shows that the polymer and the quantum dots have a real affinity for each other, created by the presence of the tetrahydro- $4 H$-thiopyran-functionalized side chains. 


\section{Experimental Section}

General: Unless specified otherwise, all reactions were carried out in a $\mathrm{N}_{2}$ atmosphere. Before use, THF was distilled from sodium/benzophenone ketyl radical, chloroform from calcium chloride and $4 \AA$ molecular sieves, and acetonitrile from calcium hydride. Methanol and $N, N$-dimethylformamide (DMF) were dried on molecular sieves ( $3 \AA$ for methanol and $4 \AA$ for DMF). All solvents used in experiments involving CdTe quantum dots were degassed in advance by boiling them in vacuo for $3 \times$ 5 min.

NMR spectra were recorded on a Bruker AC300 spectrometer $\left({ }^{1} \mathrm{H}\right.$ NMR $300.133 \mathrm{MHz} ;{ }^{13} \mathrm{C}$ NMR $75.47 \mathrm{MHz}$ ) in $\mathrm{CDCl}_{3}$. Chemical shifts (ppm) are given downfield from TMS. FT-IR spectra (neat samples) were acquired with a Perkin-Elmer System 2000 spectrometer by the ATR technique. Analytical size-exclusion chromatography (SEC) was performed with a Jordi Gel DVB Mixed Bed column equipped with a Thermo Separation Product Series 200 pump and a UV/Vis detector $(\lambda=460 \mathrm{~nm})$. Molecular weight distributions are given relative to polystyrene standards. Solution UV/Vis absorption spectra were taken on a Cary $1 \mathrm{UV} / \mathrm{Vis}$ spectrophotometer in transmission mode. UV/Vis absorption spectra of polymer films, cast from chloroform solution onto quartz substrates, were measured on a Cary 5 UV/Vis-NIR spectrophotometer in transmission mode. Fluorescence spectra were taken on a Spex Fluorolog 2 apparatus. Chloroform solutions were measured in right-angle and films on quartz cast from chloroform solution were measured in front-face geometry. For all measurements in chloroform solution the absorption at $\lambda_{\text {exc }}$ was below 0.1 .

Fluorescence decay time curves were measured with a Pico Quant PDL 800-B laser as excitation source $\left(\lambda_{\text {exc }}=406 \mathrm{~nm}, 55 \mathrm{ps}\right.$ pulse width, $2.5-10 \mathrm{MHz}$ repetition rate). A combination of an iris and neutral density filters was used to attenuate the laser power as needed. The luminescence was collected through a focusing lens, filtered through a crossed polarizer and a combination of suitable optical cut-off filters, dispersed by a $0.1 \mathrm{~m}$ monochromator (1350 lines $\mathrm{mm}^{-1}$ grating, blazed at $500 \mathrm{~nm}$ ), and detected by a fast Hamamatsu photomultiplier tube (H5738P-01). The PMT signal was amplified by an inverting preamplifier (PAM-102-T; PicoQuant) and used as the start input for a Time Harp 200 multi-channel computer card, which was synchronized with the laser pulse by means of the stop input. The decay curves were obtained by time-correlated single-photon counting through time-to-amplitude conversion. The ratio of stop to start pulses was kept low (below 0.04:1) to ensure good statistics. The acquisition time for a full time trace was typically shorter than $60 \mathrm{~s}$. The instrument response function (IRF) was determined with a diluted suspension of silica particles (Ludox) as the scattering medium and the same experimental conditions as for the fluorescence decay measurements. The raw data were analyzed by the fluorescence decay analysis software program Fluofit 3.3 (PicoQuant). The instrumental resolution was $70 \mathrm{ps}$ and $120 \mathrm{ps}$ at $10 \mathrm{MHz}$ and $2.5 \mathrm{MHz}$ repetition rate, respectively ( $10 \%$ of the IRF FWHM).

Photoinduced absorption spectra were recorded as described in reference [50]. A mechanically modulated $\mathrm{CW} \mathrm{Ar}^{+}$laser pump beam $(488 \mathrm{~nm}, 275 \mathrm{~Hz})$ was used as excitation source and the probe light (with energies between 0.35 and $3.0 \mathrm{eV}$ ) was produced by a tungsten-halogen lamp. The change in transmission through the sample $(\Delta T)$ was recorded with a phase-sensitive lock-in amplifier after dispersion by a triple-grating monochromator and detection with $\mathrm{Si}$, InGaAs, and cooled InSb detectors. The photoinduced absorption $-\Delta T / T$ was calculated from the change in transmission and was corrected for the photoluminescence, which was recorded in a separate scan. Samples were held in quartz cuvettes with a path length of $1 \mathrm{~mm}$ and measurements were performed at room temperature.

Cyclic voltammetry was performed using an EG\&G Potentiostat/Galvanostat Model $263 \mathrm{~A}$ in THF containing $0.1 \mathrm{~m}$ tetrabutylammonium hexafluorophosphate as supporting electrolyte at a scanning rate of $100 \mathrm{mV} \mathrm{s}^{-1}$. Redox potentials were determined relative to an $\mathrm{Ag} / \mathrm{AgCl}$ reference electrode and were referenced to SCE by measuring the oxidation potential of the $\left[\mathrm{FeCp}_{2}\right] /\left[\mathrm{FeCp}_{2}\right]^{++}$couple $\left(0.54 \mathrm{~V}\right.$ versus $\left.\mathrm{SCE}^{[59]}\right)$.
The $\mathrm{Ag} / \mathrm{AgCl}$ reference electrode was prepared as described in reference [60].

2,5-Dibromo-3-thiophenecarboxaldehyde (2): This synthesis was carried out as much as possible in the absence of light. To a solution of 3-thiophenecarboxaldehyde $(4.50 \mathrm{~g}, 40.1 \mathrm{mmol})$ in chloroform/acetic acid (1:1 $\mathrm{v} / \mathrm{v} ; 140 \mathrm{~mL}) \quad N$-bromosuccinimide (NBS) $(15.65 \mathrm{~g}, 88.0 \mathrm{mmol})$ was added, after which the reaction mixture was stirred at room temperature for four days. To complete bromination at both thiophene $\alpha$ positions, additional aliquots of NBS (17.73 g, $99.6 \mathrm{mmol}$ in total) and chloroform/ acetic acid $(1: 1 \mathrm{v} / \mathrm{v} ; 330 \mathrm{~mL}$ in total) were added in five parts. Subsequently, water $(75 \mathrm{~mL})$ was added. After extraction with chloroform $(3 \times$ $75 \mathrm{~mL})$ the combined organic layers were washed with $\mathrm{NaOH}(1 \mathrm{~m} ; 2 \times$ $100 \mathrm{~mL})$ and water $(2 \times 100 \mathrm{~mL})$. The organic phase was dried over $\mathrm{MgSO}_{4}$ and the remaining solvent was removed under reduced pressure. The crude product was recrystallized from hexane, yielding brown crystals $(3.93 \mathrm{~g}, 14.6 \mathrm{mmol}, 36 \%)$. ${ }^{1} \mathrm{H}$ NMR: $\delta=9.80(\mathrm{~s}, 1 \mathrm{H} ; \mathrm{CHO})$, $7.34 \mathrm{ppm}(\mathrm{s}, 1 \mathrm{H}$; aromatic $\mathrm{H}) ;{ }^{13} \mathrm{C}$ NMR: $\delta=183.1,139.2,128.6,124.2$, 113.3 ppm; IR: $\tilde{v}=3308,3099$, 2871, 2857, 2116, 1821, 1749, 1666, 1644, $1520,1435,1370,1353,1252,1174,1139,1013,971,912,832,735,684$, $667 \mathrm{~cm}^{-1}$

4-[(2,5-Dibromo-3-thienyl)hydroxymethyl]tetrahydro-4 $H$-thiopyran-4carboxylic acid (3): A slightly modified literature procedure was employed. ${ }^{[39]}$ To a solution of diisopropylamine $(2.72 \mathrm{~g}, 26.9 \mathrm{mmol})$ in THF $(150 \mathrm{~mL})$, cooled to $-40^{\circ} \mathrm{C}, n$-butyllithium $(16.6 \mathrm{~mL}$ of a $1.6 \mathrm{M}$ solution in hexanes, $26.6 \mathrm{mmol}$ ) was added during $20 \mathrm{~min}$. After this mixture had been stirred at $-40^{\circ} \mathrm{C}$ for $30 \mathrm{~min}$, tetrahydro- $4 H$-thiopyran-4-carboxylic acid $\mathbf{1}^{[61]}(1.98 \mathrm{~g}, 13.5 \mathrm{mmol})$ was added as a solid. The mixture was heated at $50^{\circ} \mathrm{C}$ for $2 \mathrm{~h}$ and then cooled to $-40^{\circ} \mathrm{C}$. Subsequently, 2 $(3.66 \mathrm{~g}, 13.6 \mathrm{mmol})$ was added. The reaction mixture was stirred at $50^{\circ} \mathrm{C}$ for $2 \mathrm{~h}$ and at room temperature overnight. After quenching with water $(250 \mathrm{~mL})$ the aqueous phase was washed with diethyl ether $(3 \times 200 \mathrm{~mL})$, then acidified with $\mathrm{HCl}(2 \mathrm{M}, 200 \mathrm{~mL})$. The aqueous phase was subsequently extracted with dichloromethane $(3 \times 150 \mathrm{~mL})$. The combined organic layers were dried $\left(\mathrm{MgSO}_{4}\right)$, and filtered, after which the filtrate was concentrated in vacuo. The crude product was subjected to Kugelrohr distillation $\left(T=120^{\circ} \mathrm{C}, p=1.1 \mathrm{mbar}\right)$, which furnished a brown, glasslike solid. The product contained $\mathbf{3}$ (as identified by ${ }^{1} \mathrm{H}$ NMR signals at $\delta=6.95(\mathrm{~s}, 1 \mathrm{H}$; aromatic $\mathrm{H}), 4.88 \mathrm{ppm}(\mathrm{s}, 1 \mathrm{H} ; \mathrm{CH} \alpha$ to thiophene ring) contaminated with remnants of $\mathbf{1}$ and some unidentified side-products. Yield $3.39 \mathrm{~g}$ of $35 \%$ purity $(2.9 \mathrm{mmol} \mathbf{3}, 21 \%)$ as estimated from the integrals of the ${ }^{1} \mathrm{H}$ NMR signals of 3 .

2,5-Dibromo-3-[(tetrahydro-4H-thiopyran-4-ylidene)methyl]thiophene (4): A solution of $\mathbf{3}(2.31 \mathrm{~g}$ of $35 \%$ purity, $1.9 \mathrm{mmol} \mathbf{3})$ and $N, N$-dimethylformamide dineopentyl acetal $(2.70 \mathrm{~g}, 11.7 \mathrm{mmol})$ in acetonitrile $(125 \mathrm{~mL})$ was stirred at room temperature for $45 \mathrm{~min}$ and then at reflux temperature overnight. ${ }^{[39]}$ After removal of the solvent in vacuo, the residue was dissolved in dichloromethane $(100 \mathrm{~mL})$ and washed with water $(4 \times 150 \mathrm{~mL})$. The remaining organic phase was dried $\left(\mathrm{MgSO}_{4}\right)$ and filtered. Subsequently the filtrate was concentrated in vacuo and subjected to Kugelrohr distillation $\left(T=130^{\circ} \mathrm{C}, p=0.11 \mathrm{mbar}\right)$. The distillate was recrystallized from methanol furnishing white crystals $(0.61 \mathrm{~g}, 1.7 \mathrm{mmol}$, $89 \%) .{ }^{1} \mathrm{H}$ NMR: $\delta=6.80(\mathrm{~s}, 1 \mathrm{H}$; aromatic $\mathrm{H}), 5.92(\mathrm{~s}, 1 \mathrm{H} ;=\mathrm{C}-\mathrm{H}), 2.75$ $(\mathrm{m}, 2 \mathrm{H}), 2.68(\mathrm{~m}, 2 \mathrm{H}), 2.59 \mathrm{ppm}(\mathrm{m}, 4 \mathrm{H}) ;{ }^{13} \mathrm{C} \mathrm{NMR}: \delta=143.2,138.4$, 131.1, 117.0, 110.6, 110.1, 38.6, 32.0, 30.9, $30.0 \mathrm{ppm}$; IR: $\tilde{v}=3084,2948$, 2924, 2904, 2882, 2819, 1681, 1649, 1519, 1423, 1408, 1339, 1321, 1303, 1290, 1268, 1223, 1205, 1187, 1167, 1133, 1120, 1011, 992, 973, 946, 937, $915,852,831,768,727,690,670,656 \mathrm{~cm}^{-1}$.

Poly\{3-[(tetrahydro-4H-thiopyran-4-ylidene)methyl]thiophene\} (poly(4)): The preparation was based on a procedure developed by Yamamoto et al ${ }^{[62]}$ A mixture of $\mathrm{Ni}(\operatorname{cod})_{2}(0.21 \mathrm{~g}, 0.76 \mathrm{mmol}), 1,5$-cyclooctadiene $(70 \mu \mathrm{L}, 0.57 \mathrm{mmol})$, triphenylphosphine $(0.18 \mathrm{~g}, 0.69 \mathrm{mmol})$, and 4 $(0.1942 \mathrm{~g}, 0.55 \mathrm{mmol})$ in DMF $(5 \mathrm{~mL})$ was stirred at $60^{\circ} \mathrm{C}$ for $16 \mathrm{~h}$ and subsequently at $100^{\circ} \mathrm{C}$ for $20 \mathrm{~h}$. The reaction mixture was quenched with water $(75 \mathrm{~mL})$ and extracted with chloroform $(4 \times 100 \mathrm{~mL})$. The combined organic layers were washed with water $(100 \mathrm{~mL})$, dried $\left(\mathrm{MgSO}_{4}\right)$ and filtered. After concentration of the filtrate in vacuo the residue was washed with diethyl ether $(7 \times 50 \mathrm{~mL})$. A purple powder $(0.0827 \mathrm{~g}$, $0.43 \mathrm{mmol}$ repeating units, $78 \%$ ) with $M_{\mathrm{w}}=2.7 \times 10^{3}$ and $D=1.4$ was 
obtained. The product was soluble in chloroform and partially soluble in dichloromethane and THF. ${ }^{1} \mathrm{H}$ NMR: $\delta=6.95(\mathrm{br}, 1 \mathrm{H}$; aromatic $\mathrm{H})$, 6.21 (s, ca. $0.4 \mathrm{H} ;=\mathrm{C}-\mathrm{H}), 6.11$ (s, ca. $0.6 \mathrm{H} ;=\mathrm{C}-\mathrm{H}), 2.80(\mathrm{br}, 2 \mathrm{H}$ ), $2.63 \mathrm{ppm}$ (br, $6 \mathrm{H}) ;{ }^{13} \mathrm{C}$ NMR: $\delta=119.1,118.8,38.7,32.1,30.8,30.2 \mathrm{ppm}$ (because of a poor signal-to-noise ratio the backbone ${ }^{13} \mathrm{C}$ NMR signals could not be detected); IR: $\tilde{v}=3053,2937,2900,2824,1647,1588,1550$, 1496, 1423, 1321, 1300, 1287, 1267, 1225, 1183, 1119, 1052, 1015, 966, 936, $847,828,771,736,721,691,653 \mathrm{~cm}^{-1}$; elemental analysis calcd (\%) for $\mathrm{C}_{10} \mathrm{H}_{10} \mathrm{~S}_{2}$ (194.3): C 61.81, H 5.19, S 33.00; found: C 61.80, H 5.10, S 33.11

CdTe quantum dots: These were synthesized in a glove-box under argon by a modification of the procedure reported by Wuister et al. ${ }^{[12,20]} \mathrm{Te}$ powder $(<250 \mu \mathrm{m}$ particle size, $0.16 \mathrm{~g}, 1.3 \mathrm{mmol})$ was added to a solution of dimethylcadmium $(0.26 \mathrm{~g}, 1.8 \mathrm{mmol})$ in TOP $(20 \mathrm{~mL})$ and DDA $(15 \mathrm{~g})$, heated to $50^{\circ} \mathrm{C}$. The reaction mixture was stirred at $180^{\circ} \mathrm{C}$ and the reaction progress was monitored by measuring the fluorescence of samples consisting of a few drops of reaction mixture diluted in toluene $(10 \mathrm{~mL})$ under UV irradiation $(365 \mathrm{~nm})$. The synthesis was stopped at the moment that a sample taken from the reaction mixture showed bright orange to red fluorescence. The quantum dots had an average diameter of $4.0 \mathrm{~nm}$, as estimated from the first absorption maximum of the CdTe quantum dots, which is near $620 \mathrm{~nm} .{ }^{[1]}$

Complexation experiments: The maximum coverage of CdTe quantum dots by poly(4) was estimated from the area occupied by a repeating unit (27.7 $\AA^{2}$ as estimated from an MM2 calculation in Chem3D Pro ${ }^{[63]}$ ) and the area of a completely stripped quantum dot of known radius. The number of quantum dots in a sample was calculated under the assumption that a quantum dot consists of a $\mathrm{Te}^{2-}$ lattice with the $\mathrm{Cd}^{2+}$ ions occupying the voids. This is justified by the ionic radius of $\mathrm{Te}^{2-}\left(2.11 \AA^{[64]}\right)$ being much larger than that of $\mathrm{Cd}^{2+}\left(0.97 \AA^{[64]}\right)$. Thus, from the number of $\mathrm{Te}^{2-}$ ions fitting in a quantum dot of known radius and the amount of CdTe quantum dot material used (by taking a given amount from a dispersion of known concentration), the numbers of quantum dots, and of CdTe units, were obtained.

Before exposure to poly(4), an aliquot of CdTe quantum dots $(8 \mathrm{~mL}$ of a dispersion in TOP/DDA with a CdTe unit concentration of $0.036 \mathrm{M}$ ) was precipitated into methanol $(70 \mathrm{~mL})$. When the precipitate had settled, the supernatant was decanted. The precipitate was dispersed in chloroform $(8 \mathrm{~mL})$ and again precipitated into methanol $(70 \mathrm{~mL})$. After centrifugation ( $2400 \mathrm{rpm}$ for $15 \mathrm{~min}$ ) the supernatant was decanted and the precipitate was dispersed in chloroform $(8 \mathrm{~mL})$. For photophysical investigation of a CdTe/poly(4) mixture, a system was prepared containing CdTe quantum dots and poly(4) in a ratio of 9:1 (mol CdTe units per mol poly(4) repeating units). This implies that the occupation of the quantum dot surface by poly(4) was approximately $40 \%$ at most. It should be realized that the actual coverage by poly(4) was less than the maximum, since TOP remained attached to the quantum dot surface. Mixed systems were stored in an inert atmosphere for at least $2 \mathrm{~h}$ before measurement to allow full equilibration of the complexation process

\section{Acknowledgements}

The authors gratefully acknowledge the Netherlands Organization for Scientific Research, Priority Program of Materials (NWO-PPM), for financial support of this work.

[1] D. S. Ginger, N. C. Greenham, Phys. Rev. B 1999, 59, 10622

[2] N. C. Greenham, X. Peng, A. P. Alivisatos, Phys. Rev. B 1996, 54, 17628.

[3] N. C. Greenham, X. Peng, A. P. Alivisatos, Synth. Met. 1997, 84, 545.

[4] B. S. Ong, Y. Wu, P. Liu, S. Gardner, J. Am. Chem. Soc. 2004, 126, 3378.

[5] K. Yoshino, Y. Kawagishi, S. Tatsuhara, H. Kajii, S. Lee, M. Ozaki, Z. V. Vardeny, A. A. Zakhidov, Superlattices Microstruct. 1999, 25, 325 .
[6] A. Shik, S. Yu, E. Johnson, H. Ruda, E. H. Sargent, Solid-State Electron. 2002, 46, 61 .

[7] M. C. Schlamp, X. Peng, A. P. Alivisatos, J. Appl. Phys. 1997, 82, 5837.

[8] V. L. Colvin, M. C. Schlamp, A. P. Alivisatos, Nature 1994, 370, 354.

[9] S. Coe, W.-K. Woo, M. Bawendi, V. Bulovic, Nature 2002, 420, 800.

[10] S. Chaudhary, M. Ozkan, W. C. W. Chan, Appl. Phys. Lett. 2004, 84, 2925.

[11] W. W. Yu, L. Qu, W. Guo, X. Peng, Chem. Mater. 2003, 15, 2854.

[12] S. F. Wuister, F. van Driel, A. Meijerink, Phys. Chem. Chem. Phys. 2003, 5, 1253.

[13] D. V. Talapin, S. Haubold, A. L. Rogach, A. Kornowski, M. Haase, H. Weller, J. Phys. Chem. B 2001, 105, 2260.

[14] P. Reiss, J. Bleuse, A. Pron, Nano Lett. 2002, 2, 781.

[15] C. B. Murray, D. J. Norris, M. G. Bawendi, J. Am. Chem. Soc. 1993, 115,8706 .

[16] E. Kucur, J. Friegler, G. A. Urban, T. Nann, J. Chem. Phys. 2003, $119,2333$.

[17] L. Bakueva, S. Musikhin, M. A. Hines, T.-W. F. Chang, M. Tzolov, G. D. Scholes, E. H. Sargent, Appl. Phys. Lett. 2003, 82, 2895.

[18] A. A. Bol, A. Meijerink, J. Phys. Chem. B 2001, 105, 10203.

[19] A. A. Bol, A. Meijerink, J. Phys. Chem. B 2001, 105, 10197.

[20] S. F. Wuister, I. Swart, F. van Driel, S. G. Hickey, C. de Mello Donegá, Nano Lett. 2003, 3, 503.

[21] J. Li, X. Hong, Y. Liu, D. Li, Y. A. Wang, J. Li, Y. Bai, T. Li, Adv Mater. 2005, 17, 163.

[22] H. Bao, Y. Gong, Z. Li, M. Gao, Chem. Mater. 2004, 16, 3853.

[23] A. A. Bol, R. van Beek, A. Meijerink, Chem. Mater. 2002, 14, 1121.

[24] S. F. Wuister, A. Meijerink, J. Lumin. 2003, 102-103, 338.

[25] K. Prabakar, S. K. Narayandass, D. Mangalaraj, Physica B 2003, 328, 355.

[26] V. I. Levchenko, V. N. Yakimovich, L. I. Postnova, V. I. Konstantinov, V. P. Mikhailov, N. V. Kuleshov, J. Cryst. Growth 1999, 198/199, 980.

[27] N. Gaponik, D. V. Talapin, A. L. Rogach, A. Eychmüller, H. Weller, Nano Lett. 2002, 2, 803.

[28] N. Gaponik, D. V. Talapin, A. L. Rogach, K. Hoppe, E. V. Shevchenko, A. Kornowski, A. Eychmüller, H. Weller, J. Phys. Chem. B 2002, 106, 7177.

[29] T. Tsuruoka, K. Akamatsu, H. Nawafune, Langmuir 2004, 20, 11169.

[30] A. van Dijken, J. J. A. M. Bastiaansen, N. M. M. Kiggen, B. M. W. Langeveld, C. Rothe, A. Monkman, I. Bach, P. Stössel, K. Brunner, J. Am. Chem. Soc. 2004, 126, 7718 .

[31] J. Liu, E. N. Kadnikova, Y. Liu, M. D. McGehee, J. M. J. Fréchet, J. Am. Chem. Soc. 2004, 126, 9486.

[32] J. Liu, T. Tanaka, K. Sivula, A. P. Alivisatos, J. M. J. Fréchet, J. Am. Chem. Soc. 2004, 126, 6550.

[33] E. P. A. M. Bakkers, A. W. Marsman, L. W. Jenneskens, D. Vanmaekelbergh, Angew. Chem. 2000, 112, 2385; Angew. Chem. Int. Ed. 2000, 39, 2297.

[34] Y.-V. Kervennic, J. M. Thijssen, D. Vanmaekelbergh, R. Dabirian, L. W. Jenneskens, C. A. van Walree, H. S. J. van der Zant, Angew. Chem. 2006, 118, 2602; Angew. Chem. Int. Ed. 2006, 45, 2540.

[35] M. Poot, E. Osorio, K. O’Neill, J. M. Thijssen, D. Vanmaekelbergh, C. A. van Walree, L. W. Jenneskens, H. S. J. van der Zant, Nano Lett. 2006, 6, 1031.

[36] A. W. Marsman, R. W. A. Havenith, S. Bethke, L. W. Jenneskens, R. Gleiter, J. H. van Lenthe, Eur. J. Org. Chem. 2000, 2629.

[37] A. W. Marsman, R. W. A. Havenith, S. Bethke, L. W. Jenneskens, R. Gleiter, J. H. van Lenthe, M. Lutz, A. L. Spek, J. Org. Chem. 2000, $65,4584$.

[38] T. Rajh, O. I. Micic, A. J. Nozik, J. Phys. Chem. 1993, 97, 11999.

[39] F. J. Hoogesteger, R. W. A. Havenith, J. W. Zwikker, L. W. Jenneskens, L. W. Kooijman, N. Veldman, A. L. Spek, J. Org. Chem. 1995, $60,4375$.

[40] Compound 4 was also polymerized in the presence of CdTe quantum dots. However, as indicated by photophysical studies, the quantum dots did not survive the polymerization. CdTe quantum dots 
can be neither oxidized nor reduced by $\left[\mathrm{Ni}(\operatorname{cod})_{2}\right]$ or $\mathrm{Ni}^{2+}$ (which is formed during the polymerization process), as the redox potential of the $\mathrm{Ni}^{2+} / \mathrm{Ni}$ couple $\left(-0.23 \mathrm{~V}\right.$ vs. $\mathrm{NHE},-0.47 \mathrm{~V}$ vs. $\left.\mathrm{SCE}^{[64]}\right)$ is situated between the oxidation and reduction potentials of CdTe quantum dots. ${ }^{[38,55,56]}$ However, oxidation by $\mathrm{Ni}^{2+}$ can occur after excitation of an electron in the quantum dots under the influence of light, since the conduction band edge of the quantum dots is at a higher energy level than the redox potential of the $\mathrm{Ni}^{2+} / \mathrm{Ni}$ couple (photooxidation). See: M. Wijtmans, PhD thesis, Radboud University, Nijmegen (The Netherlands), 2004.

[41] R. D. McCullough, P. C. Ewbank, in Handbook of Conducting Polymers, 2 ed. (Ed.: J. R. Reynolds), Marcel Dekker, New York, 1998, p. 225.

[42] M. Westerling, C. Vijila, R. Österbacka, H. Stubb, Chem. Phys. 2003, 286, 315 .

[43] R. van Beek, L. W. Jenneskens, A. N. Zdravkova, J. P. J. M. van der Eerden, C. A. van Walree, Macromol. Chem. Phys. 2005, 206, 1006.

[44] The comparison with PTT is made because this is one of the few polythiophenes for which cyclic voltammetry in THF solution has been reported. The photophysical properties and redox behavior of PTT resemble those of poly(3-alkylthiophene)s. See also reference [43].

[45] M. Belletête, L. Mazerolle, N. Desrosiers, M. Leclerc, G. Durocher, Macromolecules 1995, 28, 8587.

[46] J. Seixas de Melo, H. D. Burrows, M. Svensson, M. R. Andersson, A. P. Monkman, J. Chem. Phys. 2003, 118, 1550 .

[47] X. M. Jiang, R. Österbacka, C. P. An, Z. V. Vardeny, Synth. Met. 2003, 137,1465 .

[48] X. M. Jiang, R. Österbacka, O. Korovyanko, C. P. An, B. Horovitz, R. A. J. Janssen, Z. V. Vardeny, Adv. Funct. Mater. 2002, 12, 587.

[49] B. Kraabel, D. Moses, A. J. Heeger, J. Chem. Phys. 1995, 103, 5102.
[50] P. A. van Hal, M. P. T. Christiaans, M. M. Wienk, J. M. Kroon, R. A. J. Janssen, J. Phys. Chem. B 1999, 103, 4352.

[51] M. Westerling, R. Österbäcka, H. Stubb, Phys. Rev. B 2002, 66, 165220 .

[52] M. Westerling, R. Österbacka, H. Stubb, Thin Solid Films 2002, 403-404, 510.

[53] E. M. Conwell, H. A. Mizes, Phys. Rev. B 1995, 51, 6953.

[54] A. Weller, Z. Phys. Chem. (Muenchen Ger.) 1982, 133, 93.

[55] Y. Bae, N. Myung, A. J. Bard, Nano Lett. 2004, 4, 1153.

[56] S. K. Poznyak, N. P. Osipovich, A. Shavel, D. V. Talapin, M. Gao, A. Eychmüller, N. Gaponik, J. Phys. Chem. B 2005, 109, 1094.

[57] J. W. Thackeray, M. J. Natan, P. Ng, M. S. Wrighton, J. Am. Chem Soc. 1986, 108, 3570.

[58] It is not excluded that in solution ultrafast energy transfer from poly(4) to the quantum dots takes place before the charge separation. However, energy transfer was not resolved in our experiments.

[59] A. Facchetti, M.-H. Yoon, C. L. Stern, G. R. Hutchison, M. A. Ratner, T. J. Marks, J. Am. Chem. Soc. 2004, 126, 13480.

[60] J. Friedrich, M. Baumgarten, Appl. Magn. Reson. 1997, 13, 393.

[61] S. Strässler, A. Linden, Helv. Chim. Acta 1997, 80, 1528.

[62] T. Yamamoto, A. Morita, Y. Miyazaki, T. Maruyama, H. Wakayama, Z. Zhou, Y. Nakamura, T. Kanbara, Macromolecules 1992, 25, 1214.

[63] Chem3D Pro 8, Version 8.0.3, CambridgeSoft, Cambridge (USA), 2004.

[64] Handbook of Chemistry and Physics, 59th ed. (Eds.: R. J. Weast, M. J. Astle), CRC Press, Boca Raton, 1979.

Received: November 29, 2005 Revised: May 30, 2006 Published online: August 10, 2006 